\title{
Detection of Covid-19 from X-ray Images via Ensemble of Features Extraction Methods Employing Randomized Neural Networks

\author{
Ömer Faruk Ertuğrul' ${ }^{10}$, Emrullah Acar ${ }^{1}$ (D), Abdulkerim Öztekin ${ }^{1}$ (D) Erdoğan Aldemir ${ }^{1+}$ (D)
}

1* Electrical and Electronics Engineering Dept., Archit. and Eng. Faculty, Batman University. (e-mail: erdogan.aldemir@batman.edu.tr).

\section{ARTICLE INFO}

Received: May, 19. 2021

Revised: Dec., 23. 2021

Accepted: Dec., 29. 2021

\section{Keywords:}

Covid-19

neural network

textural features,

automatic diagnosis

Corresponding author: E. Aldemir

ISSN: 2536-5010 | e-ISSN: 2536-5134

DOI: https://doi.org/10.36222/ejt.1035007

\section{ABSTRACT}

Artificial intelligence-based solutions have achieved significant successes in the field of health in recent years. These solutions have been started to be used for pre-diagnosis and decision support for a virus that spreads rapidly such as COVID-19 and thus creates fear and panic among the public. They also have augmented clinical expertise and thus have great potential to mitigate the virus outbreak burden of health experts. In this context, the load of healthcare workers can be significantly reduced through the help of an automatic diagnosis system of a high number of patients who apply to healthcare organizations with suspicion of disease. In this study, a machine-learning automatic diagnosis system exploiting x-ray images is proposed to detect diseases caused by COVID-19. The proposed system employs powerful texture features (Histogram of Oriented Gradients, Law's Texture Energy Measure, Gabor Wavelet Transform, Gray Level Co-Occurrence Matrix, and Local Binary Pattern) for the $\mathrm{x}$-ray images to train a randomized neural network, a fast network, to establish a robust and fast diagnosis process for the virus. This study has raised the thesis that the mentioned image texture features extracted from the virus patients' images contain determinative indicators in two-dimensional space that make it possible to diagnose the disease. The proposed system contributes to the literature by using the tissue properties of $\mathrm{x}$-ray images for the diagnosis of the virus. The disease is detected with an accuracy of $\% 100$ utilizing Law's Texture Energy Measure features and randomized neural network approach.

\section{INTRODUCTION}

Humanity faces a major disaster rarely seen in history due to coronavirus disease, nowadays. This highly pathogenic coronavirus 2019 (COVID-19) is the new type of the coronavirus family, which is also called SARS-CoV-2 standing for severe acute respiratory syndrome coronavirus 2 [1]. To date, the COVID-19 has infected over one hundred million humans worldwide, with over 2 million deaths, and still continues threatening our lives [2]. The clinical studies have shown that most of the COVID-19 patients suffer from fever, cough, and shortness of breath, and other symptoms may include abdominal pain, muscle pain, diarrhea, sore throat, chills, sputum production, and loss of taste and smell [3].

Even though the spread of the virus may be possible before the appearance of symptoms, in symptomatic cases it is most contagious and symptoms may appear between 2-14 days after exposure to the virus [4]. Older adults and people who have severe medical conditions seem to be at higher risk [5] and need to take extra precautions [6]. Unfortunately, there is currently no specific antiviral treatment method or vaccine therapy for COVID-19, however, there are many clinical trials evaluating potential therapies.

To combat the spread of COVID-19 disease, effective screening of patients and emergency medical response for infected patients is vital. The standard screening method of diagnosis is by reverse transcription-polymerase chain reaction (RT-PCR) test using nasopharyngeal swabs [7], [8]. Although the transcription-polymerase chain reaction (RT-PCR) test is the most common and reliable method for COVID-19 detection, it is a time-consuming process, laborious and complex in means of application [9]. The epidemiological history of the disease, clinical symptom analysis, positive pathogenic tests, and chest radiography (i.e., CT and X-ray) can be used in the diagnosis of COVID-19. In most of the COVID19 cases, radiographic images have similar features in the early stage and pulmonary consolidation in the late stage [10]. A comparison of chest CT to RT-PCR test in the diagnosis of COVID-19 has been given by the study of Fang et al., which reports up to $98 \%$ sensitivity chest CT [11].

Today, artificial intelligence (AI) based analyses are widely used in the diagnosis of a variety of types of tumor and cancer detection. A number of deep learning and machine learningbased methods have been employed for the detection and screening of COVID-19 on the CT dataset, which is given in [12], [13]. There are several studies in the literature using AI and/or deep learning-based detection techniques from chest CT scans for the analysis and pre-diagnosis of COVID-19 cases, prior to applying PCR or pathogenic tests and thus saving significant time for further treatments [14], [15]. The CT 
images of chests is known to be a very effective imaging method in the diagnosis of lung-related illness; however, chest $\mathrm{x}$-ray imaging is more common in terms of availability, cost, and processing time [16]. One of the best ways to combat this epidemic is to turn the time in our favor by making the fastest diagnosis. The $\mathrm{x}$-ray imaging is almost available even in all rural regions and most ambulatory care facilities and plays a vital role in clinical treatments and epidemiological studies [17]. Thus, the pandemic has pushed the health system to develop a telemedicine-integrable solution [18]. It is frequently used in the diagnosis of pneumonia, lung irritation/inflammation, swellings, distended/enlarged lymph nodes, and abscesses. While a typical x-ray imaging is very helpful in the early screening of suspicious cases, it may sometimes cause confliction as images of positive COVID-19 cases are similar to images of other viral cases of pneumonia that cause difficulties in distinguishing it from other cases. It, therefore, requires a radiologist to be available to perform the task, and this may take a considerably valuable time when the epidemic rapidly spreads and healthcare professionals and other resources are scarce [19]. A misdiagnosis of a true positive COVID-19 case will cause a very crucial risk, as well as a misdiagnosis of the non-positive case, will cause unnecessary effort, cost, and congestion in the current situation where hospitals are overloaded. It is, therefore, necessary to develop and design an AI-based automatic diagnosis system to shorten the analysis time and significantly speed up the screening for medical professionals.

The rest of the paper has been organized as follows: the details of the image dataset and the employed methodology are expressed in Section 2. The results and discussion are given in Section 3 and Section 4, respectively. Finally, Section 5 concludes the paper.

\section{Material and Methods}

\subsection{Data Collection and Image Pre-processing}

The data used in this study is attained from the Kaggle's Chest X-ray Images (Pneumonia) dataset [20]. The data set consists of 50 total $\mathrm{x}$-ray images (25 of patients are diagnosed as COVID-19 positive and 25 of patients are healthy). Sample images for normal and Covid-19 x-ray are indicated in Figure 1 .

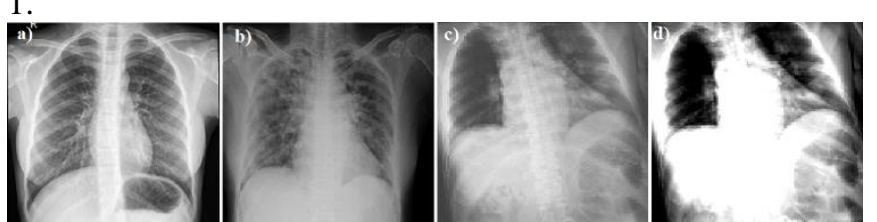

Figure 1. a) healthy patient b) Covid-19 patient c) low contrast x-ray d) highcontrast $\mathrm{x}$-ray

Each of $x$-ray images is resized as $256 \times 256$. Then, these images are converted to 8-bit (unsigned integer) resolution monochrome images. Then, the low-contrast of each gray scale image is adjusted into high-contrast image intensity values as shown in Figure 1. The multi-feature extraction methods, which consist of Histogram of Oriented Gradients (HOG), Law's Texture Energy Measurement (TEM), Gabor Wavelet Transform (GWT), Gray Level Co-Occurrence Matrix (GLCM), and Local Binary Pattern (LBP), are implemented to the attained pre-processed gray scale images and mentioned methods are elucidated in the following sub-section.

\subsection{Feature Extraction}

\subsubsection{Histogram of Oriented Gradients}

Histogram of Oriented Gradients (HOG) is a gradient-based feature extraction method that is firstly suggested in the research of [21]. It explains an image as a series of local histograms and each local histograms is the dispersion of the occurrences number of gradients in specified directions computed in a particular field described as a cell upon the image [22]. The stage of feature extraction with the HOG algorithm is accomplished in three steps, which are the computation of gradient, computation of histogram, and block normalization.

\section{The Computation of Gradient}

The gradient values in horizontal and vertical directions of each point on the image are computed with the following equations. Here, Sobel filters are mostly employed to measure these components.

$$
\begin{aligned}
& f_{x}(i, j)=I(i+1)-I(i-1, j) \\
& f_{y}(i, j)=I(i, j+1)-I(i, j-1)
\end{aligned}
$$

Here, the expression $I(i, j)$ points out the brightness at the spatial coordinates $(i, j)$. The magnitude of the computed gradients $\mathrm{M}(\mathrm{i}, \mathrm{j})$ and the direction of the gradient $\phi(i, j)$ are indicated via the following equation (3) and equation (4).

$$
\begin{aligned}
& M(i, j)=\sqrt{\left\{f_{i}(i, j)^{2}+f_{j}(i, j)^{2}\right\}} \\
& \phi(i, j)=\arctan \left(\frac{f_{i}(i, j)}{f_{j}(i, j)}\right)
\end{aligned}
$$

\section{The Computation of Histogram (Experimental Distribution)}

While computing the histogram, $\phi(i, j)$ can be categorized into identical regions in the range of $0^{\circ}$ to $180^{\circ}$ or $0^{\circ}$ to $360^{\circ}$ angles, based on the implementation. In our study, nine gradient regions in different directions are picked up between $0^{\circ}-$ $180^{\circ}$ with angles of $20^{\circ}$. The gradient dimension of each pixel in the cell is deployed to histogram regions on the basis of the angle it has, as indicated in Figure 1. This deployment is commonly carried out by linear and trilinear interpolation approaches [21]. The linear interpolation is utilized in our study.

\section{Block Normalization}

The main histogram is acquired by merging the entire sub histograms formed in a block and it is normalized using the following equation (5):

$v=\left(\frac{V_{m}}{\sqrt{\left\|V_{m}\right\|^{2}}+1}\right)$

Here, $\mathrm{v}$ and $\mathrm{Vm}$ represent the normalized HOG feature vector and the main histogram vector of a block, respectively. In our study, histogram regions are constituted by computing the unsigned gradients on rectangular cells (R-HOG) [23] and L2-Norm is employed for normalization. Moreover, the whole image is 125 split up into $3 \times 3$ rectangular cells, and a local histogram is formed from each cell. After that, nine histogram regions per cell $\left(180^{\circ} / 20^{\circ}=9\right)$ are integrated with nine local histograms, and a feature vector with a length of $(9 \times 9=$ $81)$ is generated. Finally, these vectors are used as inputs of the classification stage. 


\subsubsection{Laws Texture Energy Measure}

Laws Texture Energy Measure (TEM) method gathers preset one-dimensional kernels into diverse convolution filters. Then, one-dimensional filters with vector length $1=5$ : L5

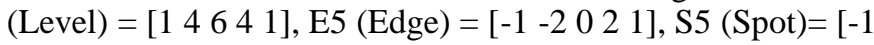

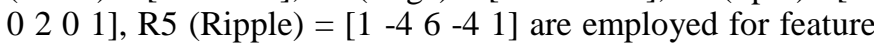
extraction. There, L5 offers the center-weighted local average. E5 replies to step edges in columns and rows. S5 detects points and R5 identifies fluctuations. If a column vector is multiplied by a row vector of the same length, a filter of size $1 \times 1$ is attained. Thus, different size filters are attained employing diverse rows and columns. These filters are then convolved with the image in order to extract texture features from this image and hence the attained new image is called "energy image". In the end, feature vectors are constituted by taking the statistical values (e.g., entropy, mean, standard deviation) of the obtained energy images. The mathematical expression of the texture energy map Em for filter $m$ is stated in Equation 6.

$$
\sum_{m}(a, b)=\sum_{j=b-7}^{b+7} \sum_{i=a-7}^{a+7} F_{m}(i, j)
$$

Here, Fm(i, j), (i, j), and (a, b) represent the mth-filtered image at pixel $(i, j)$, the sizes of the filtered image, and the sizes of the energy map, respectively. Finally, first-order statistic (i.e., mean) is implemented to the energy map to form a feature vector and sixteen parameters are computed as inputs of the classification stage from each image.

\subsubsection{Gabor Wavelet Transform}

Gabor wavelets are immensely similar to the visual system of human-based on their frequency and orientation characteristics. These wavelets compose a perfect filter for both orientation and spatial localization. A complex Gabor wavelet filter can be described as the product of a complex sinusoidal and a Gaussian kernel. A two-dimensional GWT can be stated by the convolution of the image $\mathrm{I}(\mathrm{a}, \mathrm{b})$ as shown in Equation 7 [24]:

$J(a, b)=\iint I\left(a^{\prime}, b^{\prime}\right) g\left(a-a^{\prime}, b-b^{\prime}\right) d a^{\prime} d b^{\prime}$

Here, the $g f(a, b)$ function illustrates the Gabor filter as:

$g f(a, b ; \lambda, \varphi, \sigma, \gamma)=\exp \left(\frac{a_{1}^{2}+\gamma^{2}}{2 \sigma^{2}}\right) \exp \left(j\left(2 \pi \frac{a_{1}}{\lambda}+\psi\right)\right)$

$a_{1}=\operatorname{acos} \varphi+b \sin \varphi$ and $a_{1}=\operatorname{acos} \varphi+b \sin \varphi$

Here, $\phi$ and $\lambda$ correspond to the angular orientation of the Gabor function and the wavelength scale of the cosine parameter, respectively. And $\psi$ and $\gamma$ express the phase offset value and the spatial view angle, respectively. The statistical parameters are computed from the GWT that are standard deviation $(\sigma)$, mean $(\mu)$, and entropy $(\epsilon)$. Assume that $I(i, j)$ represents the GWT matrix of each gray scale image with the size of $\mathrm{m} \times \mathrm{n}$, then these parameters are computed as shown in Equation 9.

$\mu_{i j}=\frac{1}{m n} \sum_{i}^{m} \sum_{j}^{n} I_{i j}$

$\sigma_{i j}=\frac{1}{m n} \sum_{i}^{m} \sum_{j}^{n}\left(I_{i j}-\mu_{i j}^{2} I_{i j}\right)$

$\epsilon_{i j}=-\sum_{i, j} I(i, j) \log \left(I_{i j}\right)$
After calculating three statistical parameters for each wavelet, the statistical variables obtained from eight wavelets (two scales and four angular orientations) of each image are added sequentially and $(3 \times 8=24)$ parameters are created to form the feature vector of each gray scale image.

\subsubsection{Gray Level Co-Occurrence Matrix}

Gray Level Co-Occurrence Matrix (GLCM) describes the relationship amid the reference pixel and adjacent pixel [25]. A co-occurrence matrix is clarified as being the distribution of cooccurring pixel values, at a predetermined offset $(\Delta \mathrm{a}, \Delta \mathrm{b})$. The number of columns and rows of a GLCM matrix is equal to the 145 gray level numbers in the image. In which, the distribution is set on the basis of the distance between the angles and pixels [26]. An image with $p$ distinct gray levels gives a $p \times p$ cooccurrence matrix for a predetermined offset. The GLCM matrix $(\mathrm{G})$ of an image $\mathrm{I}(\mathrm{k} \times 1)$ is indicated in Equation 10 .

$G_{\Delta \mathrm{a}, \Delta \mathrm{b}}=\sum_{a=1}^{k} \sum_{b=1}^{l} \begin{cases}1 & I(a, b)=i \text { and } I(a+\Delta, b+\Delta b)=j \\ 0 & \text { otherwise }\end{cases}$

Here, $(a, b),(i, j),(\Delta a, \Delta b)$ and $I(a, b)$ illustrate the spatial location in the image, the pixel values, the offset values, and the pixel value at pixel $(a, b)$, respectively. The $G$ matrix can also be described in terms of an angle $\theta$ and distance $d$ instead of the offset values $(\Delta \mathrm{a}, \Delta \mathrm{b})$ and $\mathrm{I}(\mathrm{a}, \mathrm{b})$. Here, $\theta$ angles are used commonly as $0 \circ, 45^{\circ}, 90^{\circ}$, and $135^{\circ}$ [27]. In this study, 22 GLCM parameters are employed which consist of autocorrelation, contrast, correlation, cluster prominence, cluster shade, difference entropy, difference variance, dissimilarity, energy, entropy, homogeneity, information measure of correlation $1 \& 2$, inverse difference normalization, inverse difference moment normalization, maximum probability, sum average, sum entropy, the sum of squares and sum variance. Mathematical statements of these parameters are given in detail in [28].

\subsubsection{Local Binary Pattern}

Micro-texons are sought for on the basis of the relation among the pixels and their neighbors by Local Binary Pattern (LBP) [29]. A binary number is then assigned by comparing a central pixel by its neighbors [30]. After all comparisons, a set of binary numbers is attained and these numbers are transformed to decimal numbers that will be employed instead of these pixels [30]. The decimal number corresponds to the textural information regarding the relationship among the central pixel and its neighbors. Thus, each of them indicates a distinct pattern. The statement of Local Binary Pattern (LBP) [L(x)] for each pixel is indicated as [31].

$$
\begin{aligned}
& t=I\left(x_{i}\right)-I(x) \\
& L(x)=\sum_{i=0}^{p} R(t)^{2}(i-1) \\
& R(t)= \begin{cases}1 & t \geq 0 \\
0 & t>0\end{cases}
\end{aligned}
$$

Here, $x, x i$, and I(.) represents the central pixel, neighboring pixel at ith, and the gray-scale image, respectively. Eventually, a histogram is composed of the attained decimal numbers that are between 0 and 255 [33]. Moreover, it is observed that uniform patterns might be used in place of an overall histogram that has 256 distinct values. The uniform patterns have at the best bitwise passage as ( 0 to 1 or 1 to 0$)$.

\subsubsection{Randomized Neural Network}

Randomized Neural Network (RNN) is a robust machine learning method for feed-forward neural networks (FFNN), 
which has a single hidden layer. It can be utilized for classification and regression purposes. Input weights and bias parameters are not required to be set in contrast to other usual feed-forward network implementations. Therefore, these parameters can be chosen as random, and the output weights can be analytically ascertained. This situation allows RNN to be easy and fast in data processing [32] and ensures a fine generalization achievement for a single FFNN [33]. Compared to the other known gradient-based learning algorithms, RNN has several advantages like the potential of reaching the minimum training error, operating with non-differentiable activation functions, and employing a single hidden layer [34]. Moreover, the number of observations is more than the number of neurons in the RNN hidden layer [35]. The RNN output $\mathrm{y}$ can be computed via Equation 14 [33].

$$
y=\sum_{j=1}^{m} \rho_{j} G\left(\sum_{i=1}^{n} W_{i j} \cdot x_{i}+b_{j}\right)
$$

Here, $x, n, m, W i j, \rho j$, bj and $G($.$) express the input,$ neuron numbers of the input, neuron numbers of the hidden layer, the input weights, the output weights, the biases of the neurons, and the activation function, respectively.

\subsection{The Proposed Approach}

The block diagram of the recommended system is indicated in Figure 2. First, a dataset, which consists of $50 \mathrm{x}$-ray images (25 COVID-19 positives and 25 normal cases) are obtained

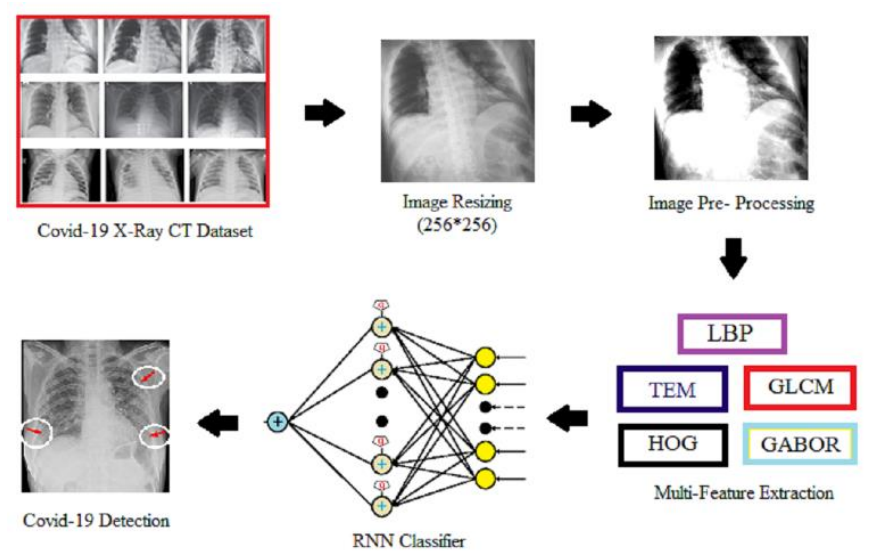

Figure 2. A block diagram, which summarizes all stages of the Covid-19 detection from x-ray images.

from the Kaggle. Second, whole $\mathrm{x}$-ray images are resized as $256 \times 256$ and these images are transformed to 8-bit resolution gray scale images. After the transformation phase, the lowcontrast of each gray scale image is set into high-contrast image intensity values. The multi-feature extraction methods, namely HOG, TEM, GWT, GLCM, and LBP, are then applied to the pre-processed images.

Finally, the attained feature vectors for each image are employed as inputs of the RNN classifier to determine whether the existence of the virus. In the training and testing stage of RNN, 10-fold cross-validation is implemented and 16 different activation functions (1-sigmoid, 2-sinusoidal, 3-radial-basis, 4hard-limit, 5-symmetric hard-limit, 6-saturating linear, 7tangent sigmoid, 8- triangular-basis, 9-positive linear, 10-pure linear, 11-cosinusoidal, 12-log sigmoid, 13-saturating linear, 14-hyperbolic tangent, 15-Gaussian, 16-multi quadratic) with the different number of hidden neurons (NHN) is chosen to determine optimal parameters. Moreover, the testing accuracies vs. NHN for all activation functions (AF) are given in the Results and Discussion section. Here, the testing accuracy is computed as:

$$
\text { Testing Accuracy }(\%)=\frac{T P+T N}{T P+T N+F P+F N} \times 100
$$

Here, TP, FN, FP, and TN illustrate the number of true positives, false negatives, false positives, and true negatives, respectively.

\section{EXPERIMENTS}

In this section, the total of $50 \mathrm{x}$-ray images (25 COVID-19 positives and 25 normal) with different resolutions are resized as $256 \times 256$ and they are converted to 8 -bit resolution gray scale images. After that, the contrast of resized gray scale images is increased into high-intensity values. Then, multifeature extraction methods, which are HOG, TEM, GWT, GLCM, and LBP, are implemented to the pre-processed monochrome images and feature vectors are attained from them. As a final step, the RNN classifier that utilized the feature vectors extracted from x-ray images detects COVID-19. The methodology performances are presented individually for all feature vectors are employed in the following subsections.

\subsection{Detection of COVID-19 from X-ray Images Using HOG and RNN}

In this section, $81 \mathrm{HOG}$ features are employed as inputs of the RNN classifier, and testing accuracies vs. NHN for all activation functions (AF) are given in Table I. The performance of the system employed by the HOG feature differs according to the parameters of seven NHN and sixteen AF functions. There is not an evident linear relationship between the accuracy of diagnosis and the NHN and AF parameters. The maximum accuracy rate, which is $95 \%$, is obtained by employing AF-2 with 20 NHN.

TABLE I

Testing Accuracy (\%) for all AF with 5 different NHN using HOG features and RNN Classifier

\begin{tabular}{|c|c|c|c|c|c|c|c|c|c|c|c|c|c|}
\hline \multirow{2}{*}{ NHN } & \multicolumn{10}{|c|}{ AF } \\
\cline { 2 - 15 } & 1 & 2 & 3 & 4 & 5 & 6 & 8 & 9 & 10 & 12 & 13 & 14 & 15 \\
\hline $\mathbf{1}$ & 30 & 34 & 34 & 33 & 36 & 38 & 33 & 35 & 35 & 34 & 36 & 33 & 36 \\
\hline $\mathbf{5}$ & 73 & 66 & 61 & 35 & 33 & 66 & 76 & 71 & 69 & 64 & 78 & 76 & 74 \\
\hline $\mathbf{1 0}$ & 83 & 88 & 80 & 41 & 40 & 86 & 88 & 81 & 88 & 74 & 80 & 79 & 79 \\
\hline $\mathbf{1 5}$ & 85 & 84 & 85 & 45 & 41 & 88 & 81 & 90 & 91 & 83 & 83 & 91 & 86 \\
\hline $\mathbf{2 0}$ & 86 & $\mathbf{9 5}$ & 83 & 43 & 44 & 88 & 81 & 89 & 86 & 91 & 85 & 93 & 83 \\
\hline $\mathbf{2 5}$ & 85 & 89 & 84 & 48 & 45 & 83 & 85 & 90 & 80 & 84 & 88 & 80 & 79 \\
\hline $\mathbf{5 0}$ & 84 & 83 & 80 & 53 & 44 & 75 & 78 & 78 & 76 & 73 & 85 & 71 & 71 \\
\hline
\end{tabular}

In general, the performances for NHN 1 and 5 remain under average performance compared to other NHN. However, considering the AF parameter, there does not exist a significant difference in the performance of the system between the AF parameters employed, except the functions 4 and 5 that provide the least accuracy rate regardless of the NHN parameters. In addition, the activation functions AF-7, AF-11, and AF-16 were also applied experimentally, and they are not presented in Table I because their performances are below the other functions given in the table. 


\subsection{Detection of COVID-19 from X-ray Images Using TEM and RNN}

The proposed system employs 15 TEM features as inputs of the RNN classifier and testing accuracies vs. NHN for all activation functions (AF) are yielded in Table II. Similar to the HOG, except for functions 4 and 5, the use of other functions (AF parameters) does not make a significant difference to the performance of the system, and functions 4 and 5 provide the least accuracy rate regardless of the NHN parameters. The system achieves the highest accuracy rate, which is $100 \%$, by employing AF-7 with 10 NHN. The TEM feature achieves better accuracy of diagnosis compared to the HOG feature, in general.

The performance of the system considerably decreases for NHN 1 and 50. The decrease reaches up to nearly $50 \%$ compared to the previous accuracy rate, e.g. $88 \%$ for NHN 25 and $54 \%$ for NHN 50 both for the AF 3. Accordingly, the determination of optimality for the parameters of the system is crucial to obtain better results for diagnosis. The system employing the TEM reaches remarkable performance for diagnosis of the COVID-19, overall.

TABLE II

Testing Accuracy (\%) for all AF with 5 different NHN using TEM features andRNN Classifier

\begin{tabular}{|c|c|c|c|c|c|c|c|c|c|c|c|c|c|c|c|c|}
\hline \multirow{2}{*}{ NHN } & \multicolumn{10}{|c|}{$\mathbf{A F}$} \\
\cline { 2 - 18 } & 1 & 2 & 3 & 4 & 5 & 6 & 7 & 8 & 9 & 10 & 11 & 12 & 13 & 14 & 15 & 16 \\
\hline $\mathbf{1}$ & 36 & 40 & 34 & 36 & 32 & 32 & 36 & 34 & 42 & 36 & 36 & 40 & 36 & 36 & 32 & 30 \\
\hline $\mathbf{5}$ & 82 & 92 & 82 & 36 & 38 & 92 & 94 & 82 & 84 & 88 & 92 & 88 & 90 & 90 & 88 & 96 \\
\hline $\mathbf{1 0}$ & 92 & 88 & 86 & 38 & 38 & 88 & $\underline{\mathbf{1 0 0}}$ & 94 & 96 & 92 & 92 & 92 & 90 & 92 & 94 & 94 \\
\hline $\mathbf{1 5}$ & 94 & 96 & 88 & 40 & 38 & 90 & 92 & 94 & 88 & 86 & 96 & 94 & 82 & 90 & 88 & 92 \\
\hline $\mathbf{2 0}$ & 90 & 94 & 86 & 58 & 46 & 92 & 88 & 90 & 88 & 88 & 92 & 92 & 88 & 88 & 92 & 92 \\
\hline $\mathbf{2 5}$ & 86 & 88 & 88 & 42 & 54 & 94 & 88 & 86 & 94 & 88 & 86 & 88 & 88 & 82 & 86 & 94 \\
\hline $\mathbf{5 0}$ & 66 & 54 & 72 & 44 & 66 & 88 & 64 & 86 & 88 & 94 & 60 & 66 & 90 & 58 & 60 & 66 \\
\hline
\end{tabular}

\subsection{Detection of COVID-19 from X-ray Images Using GWT and RNN}

The system is implemented using 24 GWT features as inputs of the RNN classifier and testing accuracies vs. NHN for all activation functions (AF) are yielded in Table III. The GWT is the feature that provides knowledge about the orientation of the patterns existing in $\mathrm{x}$-ray images that may uncover the indicators for diagnosis. The performance of the GWT reaches a $96 \%$ rate for diagnosis that is the second-highest performance. A recent deep-learning classifier announces a $\% 95$ accuracy rate of diagnosis using chest Xr-ray images [36].

\section{TABLE III}

Testing Accuracy (\%) for all AF with 5 different NHN using GWT features and RNN Classifier

\begin{tabular}{|l|c|c|c|c|c|c|c|c|c|c|c|c|c|c|c|c|}
\hline \multirow{2}{*}{ NHN } & \multicolumn{10}{|c|}{$\mathbf{A F}$} \\
\cline { 2 - 16 } & 1 & 2 & 3 & 4 & 5 & 6 & 7 & 8 & 9 & 10 & 11 & 12 & 13 & 14 & 15 & 16 \\
\hline $\mathbf{1}$ & 26 & 22 & 24 & 32 & 26 & 32 & 32 & 26 & 24 & 24 & 26 & 22 & 30 & 32 & 26 & 30 \\
\hline $\mathbf{5}$ & 64 & 68 & 64 & 28 & 28 & 64 & 78 & 64 & 70 & 76 & 74 & 72 & 62 & 60 & 66 & 70 \\
\hline $\mathbf{1 0}$ & 82 & 82 & 84 & 34 & 32 & 84 & 76 & 86 & 88 & 84 & 72 & 84 & 86 & 80 & 78 & 80 \\
\hline $\mathbf{1 5}$ & 82 & 76 & 82 & 28 & 26 & 88 & 86 & 82 & 88 & 86 & 78 & 84 & 88 & 84 & 82 & 74 \\
\hline $\mathbf{2 0}$ & 92 & 84 & 84 & 26 & 28 & 92 & 90 & 90 & 88 & 92 & 92 & 92 & 86 & 94 & 86 & 80 \\
\hline $\mathbf{2 5}$ & 82 & 80 & 90 & 36 & 30 & 88 & 86 & $\mathbf{9 6}$ & 92 & 90 & 88 & 86 & 90 & 86 & 86 & 88 \\
\hline $\mathbf{5 0}$ & 60 & 58 & 48 & 28 & 32 & 86 & 66 & 82 & 92 & 88 & 58 & 68 & 94 & 60 & 62 & 68 \\
\hline
\end{tabular}

The feature provides the least accuracy rate in the case of NHN 1, 5, and 50, similar to TEM and HOG. For the remaining NHN, the systems achieve a notable accuracy rate of diagnosis.

\subsection{Detection of COVID-19 from X-ray Images Using GLCM and RNN}

For detection of the virus, 22 GLCM features are employed as inputs of the RNN classifier, and testing accuracies vs. NHN for all activation functions (AF) are given in Table IV. Overall performance of the GLCM feature remains under the TEM, HOG, and GWT.

TABLE IV

Testing Accuracy (\%) for all AF with 5 different NHN using GLCM features and RNN Classifier

\begin{tabular}{|l|c|c|c|c|c|c|c|c|c|c|c|c|c|c|c|c|}
\hline \multirow{2}{*}{ NHN } & \multicolumn{10}{|c|}{ AF } \\
\cline { 2 - 17 } & 1 & 2 & 3 & 4 & 5 & 6 & 7 & 8 & 9 & 10 & 11 & 12 & 13 & 14 & 15 & 16 \\
\hline $\mathbf{1}$ & 30 & 34 & 30 & 28 & 26 & 26 & 32 & 34 & 34 & 34 & 30 & 32 & 32 & 28 & 26 & 26 \\
\hline $\mathbf{5}$ & 62 & 78 & 62 & 34 & 38 & 70 & 54 & 46 & 60 & 58 & 58 & 64 & 62 & 58 & 62 & 66 \\
\hline $\mathbf{1 0}$ & 56 & 60 & 62 & 32 & 42 & 68 & 66 & 64 & 60 & 72 & 64 & 58 & 74 & 54 & 56 & 68 \\
\hline $\mathbf{1 5}$ & 78 & 82 & 70 & 32 & 42 & 70 & 66 & 70 & 76 & 72 & 78 & 80 & 68 & 78 & 74 & 58 \\
\hline $\mathbf{2 0}$ & 78 & 74 & 78 & 44 & 48 & 78 & 76 & 66 & 68 & 66 & 68 & 74 & 70 & 74 & 84 & 72 \\
\hline $\mathbf{2 5}$ & 76 & 78 & $\mathbf{8 8}$ & 40 & 32 & 72 & 70 & 70 & 78 & 80 & 78 & 86 & 66 & 74 & 80 & 68 \\
\hline $\mathbf{5 0}$ & 64 & 50 & 58 & 40 & 48 & 76 & 46 & 62 & 68 & 66 & 58 & 48 & 74 & 60 & 40 & 72 \\
\hline
\end{tabular}

The system performed by the GLCM reaches an $88 \%$ rate that remains under the performance of the aforementioned features for diagnosis. Not surprisingly, functions 4 and 5 provide the least diagnosis performance while the performance of other activation functions does not show a notable fluctuation.

\subsection{Detection of COVID-19 from X-ray Images Using $\angle B P$ and $R N N$}

The proposed system has been implemented with 6 LBP features that are employed as inputs of RNN classifier and testing accuracies vs. NHN for all activation functions (AF) are yielded in Table $\mathrm{V}$.

TABLE V

Testing Accuracy (\%) for all AF with 5 different NHN using LBP features and RNN Classifier

\begin{tabular}{|c|c|c|c|c|c|c|c|c|c|c|c|c|c|c|c|c|}
\hline \multirow{2}{*}{ NHN } & \multicolumn{10}{|c|}{ AF } \\
\cline { 2 - 15 } & 1 & 2 & 3 & 4 & 5 & 6 & 7 & 8 & 9 & 10 & 11 & 12 & 13 & 14 & 15 & 16 \\
\hline $\mathbf{1}$ & 28 & 26 & 30 & 38 & 28 & 30 & 36 & 32 & 28 & 24 & 28 & 26 & 32 & 38 & 28 & 32 \\
\hline $\mathbf{5}$ & 72 & 64 & 70 & 30 & 30 & 60 & 54 & 64 & 64 & 62 & 72 & 70 & 68 & 64 & 64 & 62 \\
\hline $\mathbf{1 0}$ & 64 & 74 & 66 & 44 & 36 & 56 & 60 & 68 & 60 & 64 & 62 & 72 & 70 & 66 & 68 & 58 \\
\hline $\mathbf{1 5}$ & 70 & 62 & 74 & 28 & 40 & 66 & 62 & 66 & 68 & 50 & 68 & 68 & 62 & 68 & 68 & 76 \\
\hline $\mathbf{2 0}$ & 70 & 74 & 68 & 32 & 34 & 70 & 74 & 62 & 60 & 66 & 78 & 76 & 64 & 62 & 72 & 68 \\
\hline $\mathbf{2 5}$ & 78 & 76 & 74 & 44 & 42 & 66 & 74 & 52 & 60 & 68 & 78 & 72 & 64 & 80 & 74 & 74 \\
\hline $\mathbf{5 0}$ & 74 & 68 & 68 & 38 & 50 & 62 & 80 & 72 & 72 & 64 & 80 & 58 & 54 & 84 & $\mathbf{8 6}$ & 66 \\
\hline
\end{tabular}

The proposed methodology reaches an $86 \%$ accuracy rate with parameters of the activation function number of 15 and $50 \mathrm{NHN}$. The performance of LBP remains under the other features in general. Similar to the remaining features employed by the system, the leastperformance achievement is obtained in the case of using 4 and $5 \mathrm{AF}$. 


\section{DISCUSSION}

The proposed automated COVID-19 diagnostic system has been implemented using 5 different feature extraction methods with $16 \mathrm{AF}$ and $7 \mathrm{NHN}$ parameters for a comprehensive evaluation. The best mean accuracy (\%) results using multifeature extraction methods and RNN classifiers with the aforementioned parameters are tabulated in Table VI.

\section{TABLE VI}

The best mean accuracy (\%) results with computational time (s) for all features and RNN Classifier

\begin{tabular}{|c|c|c|c|}
\hline Approach & $\begin{array}{c}\text { Comp. Time } \\
\text { (min.) }\end{array}$ & $\begin{array}{c}\text { Feature } \\
\text { Number }\end{array}$ & $\begin{array}{c}\text { Testing } \\
\text { Accuracy } \\
(\%)\end{array}$ \\
\hline HOG+RNN & $\underline{\mathbf{0 . 2 2}}$ & 81 & 95 \\
\hline TEM+RNN & 3.20 & 15 & $\underline{\mathbf{1 0 0}}$ \\
\hline GABOR+RNN & 0.83 & 24 & 96 \\
\hline GLCM+RNN & 0.25 & 22 & 88 \\
\hline LBP+RNN & 0.72 & 6 & 86 \\
\hline
\end{tabular}

In addition, analyzes are made according to the computational time parameter, which will become even more important in the case of integration of the system into an active e-health network. In terms of overall performance, the ELM feature achieved an average of $100 \%$ performance in the automatic diagnosis of the data set, leaving all other features behind. It is seen that the ELM feature, which achieved the best diagnostic performance, classified the data set in a time of 3.2 seconds, which is a longer computational load than other methods. Although ELM is seen to have a relatively higher burden in terms of computational time compared to other methods, this does not prevent real-time use of the method thanks to modern workstations and advanced systems. Other remarkable features in terms of diagnostic success are GWT and HOG. These features both achieve over $95 \%$ performance (GWT 96\% and HOG 95\%) and provide the best results in terms of computing load. The accuracy announced between $87 \%$ and $98 \%$ for binary and multi-classes images [37], respectively. Deep-learning solutions announced in [38] and [39] give over a 90\% diagnosis rate. Recent studies [40], [41] that utilize a deep learning-based framework as a support system achieves over $\% 95$ diagnosis rate. It is clear that the HOG feature has a significant advantage when considering both computational load and diagnosis performance The system performs the least but still notable diagnosis accuracies rate for the GLCM $(88 \%)$ and LBP $(86 \%)$ features. Although these two features do not impose a high computational load, their performance remains under the performance of the ELM, HOG, and GWT features. The features utilized by the system can reveal different textural features of the image due to their algorithmic and mathematical structures. One example of this is GWT, which focuses on directional tissues in chest X-ray images, revealing some indicators that other methods cannot achieve. Since the mathematical structure and algorithmic designs of other features differ, a similar conclusion with GWT can be reached characteristics properties that aid to unveil diverse structures and indicators of the $\mathrm{x}$-ray images. Therefore, an ensemble model established by utilizing diverse types of features has the potential to increase the performance of the system for COVID-19 diagnosis.

\section{CONCLUSION}

The COVID-19 pandemic, which has created great uneasiness all over the world, is a case that has not been encountered before by the modern health systems and as a result, it has brought a serious burden to the systems. In recent years, artificial intelligence-based systems have been widely used successfully in the field of health for decision support and making diagnoses. In this respect, it has the potential to significantly reduce the workload of healthcare workers by using AI-assisted systems in the preliminary diagnosis and decision-support phase of an easily transmitted virus such as COVID-19. Indeed, studies in this area have shown that artificial intelligence-based systems promise significant success.

The diagnosis and controlling of the large number of people applying to health institutions with the suspicion of a virus in a short time are serious burdens for health organizations due to the restricted time and cost of the tests. Therefore, it is clear that an artificial intelligence-based auxiliary system to be used for pre-diagnosis and decision support will make a great contribution to the health ecosystem. Motivated by this fact, an automated system based on AI that exploits multi-features extracted from x-ray images is proposed for the diagnosis of the virus, in this study. A set of two-dimensional image texture features having diverse characteristics have been used for unveiling the indicators for automatically diagnosing the cases. The TEM, GWT, LBP, GLCM, and HOG features are extracted from an $\mathrm{X}$-ray image, and the RNN decision-maker is trained with these features. These features show diverse characteristics having the ability to uncover the indicators exiting in x-ray images. These features show diagnosis performance ranging from $86 \%$ to $100 \%$. It has been observed that the most dominant feature in automatic detection of the virus is TEM and, in the applications, performed on the dataset, it is diagnosed correctly with an average performance of $100 \%$. Moreover, the various activation functions used are mathematical forms with different characteristics. Therefore, it directly affects the performance of the method in terms of physical and representation.

The fact that the system is performing a commonly used, fast, and easily accessible imaging system such as x-ray, is promising in terms of preliminary diagnosis for geographical regions where diagnostic kits are hard to access. Although the study has been carried out on a large data set, it has the potential to be further improved if the proposed system is integrated with an active health network that contains more diverse images and kinds of images such as CT or PET. Moreover, the features utilized by the proposed systems can be widened by using different textural features to improve the performance of the systems. Consequently, it has been shown that the proposed system based on AI for the diagnosis of COVID-19 using X-ray images achieved remarkable results. This proposed system, which has the potential to be developed in terms of data-set and image texture features, can be integrated into modern health systems and thus it can make significant contributions for the public heaths.

\section{REFERENCES}

[1] S. B. Stoecklin et al., "First cases of coronavirus disease 2019 (COVID19) in France: surveillance, investigations and control measures, January 2020," Eurosurveillance, vol. 25, no. 6, p. 2000094, 2020.

[2] T. T. Team, "TrackCorona," TrackCorona, 2021. 
[3] Coronavirus Disease 2019, "Symptoms of Coronavirus," 2021. .

[4] T. P. Velavan and C. G. Meyer, "The COVID-19 epidemic," Trop. Med. I\& Int. Heal., vol. 25, no. 3, p. 278, 2020.

[5] D. Flynn et al., "COVID-19 pandemic in the United Kingdom," Heal. Policy Technol., vol. 9, no. 4, pp. 673-691, 2020.

[6] CDC, "People Who Are at Higher Risk for Severe Illness." .

[7] M. E. H. Chowdhury et al., "Can AI help in screening viral and COVID19 pneumonia?," IEEE Access, vol. 8, pp. 132665-132676, 2020.

[8] L. O. Hall, R. Paul, D. B. Goldgof, and G. M. Goldgof, "Finding covid19 from chest x-rays using deep learning on a small dataset," arXiv Prepr. arXiv2004.02060, 2020.

[9] W. Wang et al., "Detection of SARS-CoV-2 in different types of clinical specimens," Jama, vol. 323, no. 18, pp. 1843-1844, 2020.

[10] M. Chung et al., "CT imaging features of 2019 novel coronavirus (2019nCoV)," Radiology, vol. 295, no. 1, pp. 202-207, 2020.

[11] Y. Fang et al., "Sensitivity of chest CT for COVID-19: comparison to RT-PCR,” Radiology, vol. 296, no. 2, pp. E115--E117, 2020.

[12] O. Gozes et al., "Rapid ai development cycle for the coronavirus (covid19) pandemic: Initial results for automated detection I\& patient monitoring using deep learning ct image analysis," arXiv Prepr. arXiv2003.05037, 2020.

[13] F. Shi et al., "Large-scale screening of covid-19 from community acquired pneumonia using infection size-aware classification (2020)," arXiv Prepr. arXiv2003.09860, 2003.

[14] S. Wang et al., "A deep learning algorithm using CT images to screen for Corona Virus Disease (COVID-19)," Eur. Radiol., pp. 1-9, 2021.

[15] Y. Li and L. Xia, "Coronavirus disease 2019 (COVID-19): role of chest CT in diagnosis and management," Am. J. Roentgenol., vol. 214, no. 6, pp. $1280-1286,2020$.

[16] J. Zhang et al., "Viral pneumonia screening on chest X-ray images using confidence-aware anomaly detection," arXiv Prepr. arXiv2003.12338, 2020.

[17] T. Cherian et al., "Standardized interpretation of paediatric chest radiographs for the diagnosis of pneumonia in epidemiological studies," Bull. World Health Organ., vol. 83, pp. 353-359, 2005.

[18] G. Ortega et al., "Telemedicine, COVID-19, and disparities: policy implications," Heal. policy Technol., vol. 9, no. 3, pp. 368-371, 2020.

[19] J. Portnoy, M. Waller, and T. Elliott, "Telemedicine in the era of COVID-19," J. Allergy Clin. Immunol. Pract., vol. 8, no. 5, pp. 14891491, 2020.

[20] Kaggle, "Chest X-ray Images (Pneumonia)," Chest X-ray Images (Pneumonia), 2021

[21] N. Dalal and B. Triggs, "Histograms of oriented gradients for human detection," in 2005 IEEE computer society conference on computer vision and pattern recognition (CVPR'05), 2005, vol. 1, pp. 886-893.

[22] E. Acar and M. S. Ozerdem, "The texture feature extraction of Mardin agricultural field images by HOG algorithms and soil moisture estimation based on the image textures," 2015, pp. 665-665, doi: 10.1109/siu.2015.7129912.

[23] O. L. Junior, D. Delgado, V. Gonçalves, and U. Nunes, "Trainable classifier-fusion schemes: An application to pedestrian detection," in IEEE Conference on Intelligent Transportation Systems, Proceedings, ITSC, 2009, pp. 432-437, doi: 10.1109/ITSC.2009.5309700.

[24] I. Buciu and A. Gacsadi, "Gabor wavelet based features for medical image analysis and classification," in 2009 2nd International Symposium on Applied Sciences in Biomedical and Communication Technologies, 2009, pp. 1-4.

[25] M.-H. Horng and J.-H. Zhuang, "Texture feature coding method for texture classification," Opt. Eng., vol. 42, no. 1, pp. 228-238, 2003.

[26] A. Emrullah, "Extraction of texture features from local iris areas by GLCM and Iris recognition system based on KNN," Eur. J. Tech., vol. 6, no. 1, pp. 44-52, 2016.

[27] D. K. Iakovidis, D. E. Maroulis, and D. G. Bariamis, "FPGA architecture for fast parallel computation of co-occurrence matrices," Microprocess. Microsyst., vol. 31, no. 2, pp. 160-165, 2007, doi: 10.1016/j.micpro.2006.02.013.

[28] R. W. Conners, M. M. Trivedi, and C. A. Harlow, "Segmentation of a high-resolution urban scene using texture operators," Comput. vision, Graph. image Process., vol. 25, no. 3, pp. 273-310, 1984.

[29] T. Ojala, M. Pietikäinen, and T. Mäenpää, "Multiresolution gray-scale and rotation invariant texture classification with local binary patterns," IEEE Trans. Pattern Anal. Mach. Intell., vol. 24, no. 7, pp. 971-987, 2002, doi: 10.1109/TPAMI.2002.1017623.

[30] R. Nosaka and K. Fukui, "HEp-2 cell classification using rotation invariant co-occurrence among local binary patterns," in Pattern Recognition, 2014, vol. 47, no. 7, pp. 2428-2436, doi: 10.1016/j.patcog.2013.09.018.

[31] T. Chakraborti and A. Chatterjee, "A novel binary adaptive weight GSA based feature selection for face recognition using local gradient patterns, modified census transform, and local binary patterns," Eng. Appl. Artif. Intell., vol. 33, pp. 80-90, 2014.

[32] Ö. F. Ertugrul and M. E. Tagluk, "A fast feature selection approach based on extreme learning machine and coefficient of variation," Turkish J. Electr. Eng. \\& Comput. Sci., vol. 25, no. 4, pp. 3409-3420, 2017.

[33] G.-B. Huang, Q.-Y. Zhu, and C.-K. Siew, "Extreme learning machine: theory and applications," Neurocomputing, vol. 70, no. 1-3, pp. 489$501,2006$.

[34] A. Öztekin and E. Erçelebi, "An efficient soft demapper for APSK signals using extreme learning machine," Neural Comput. Appl., vol. 31, no. 10 , pp. 5715-5727, 2019.

[35] M. Li and D. Wang, "Insights into randomized algorithms for neural networks: Practical issues and common pitfalls," Inf. Sci. (Ny)., vol. 382, pp. 170-178, 2017.

[36] I. Castiglioni et al., "Machine learning applied on chest x-ray can aid in the diagnosis of COVID-19: a first experience from Lombardy, Italy," Eur. Radiol. Exp., vol. 5, no. 1, pp. 1-10, 2021.

[37] T. Ozturk, M. Talo, E. A. Yildirim, U. B. Baloglu, O. Yildirim, and U. R. Acharya, "Automated detection of COVID-19 cases using deep neural networks with X-ray images," Comput. Biol. Med., vol. 121, p. 103792, 2020.

[38] M. Tougaçar, B. Ergen, and Z. Cömert, "COVID-19 detection using deep learning models to exploit Social Mimic Optimization and structured chest X-ray images using fuzzy color and stacking approaches," Comput. Biol. Med., vol. 121, p. 103805, 2020.

[39] X. He et al., "Sample-efficient deep learning for COVID-19 diagnosis based on CT scans," medrxiv, 2020.

[40] M. Fontanellaz et al., "A deep-learning diagnostic support system for the detection of COVID-19 using chest radiographs: a multireader validation study," Invest. Radiol., vol. 56, no. 6, pp. 348-356, 2021.

[41] Y. Wan, H. Zhou, and X. Zhang, "An interpretation architecture for deep learning models with the application of COVID-19 diagnosis," Entropy, vol. 23, no. 2, p. 204, 2021.

\section{BIOGRAPHIES}

Ömer Faruk Ertuğrul received the B.S., M.S., and Ph.D. degrees in electrical and electronics engineering from Hacettepe University, Dicle Univeristy, and İnönü University, respectively. $\mathrm{He}$ is currently an Associate Professor of electrical-electronics engineering with Batman University. His research interests include machine learning, signal processing and Industry 4.0.

Emrullah Acar Emrullah Acar received the B.S. degree in electrical and electronics engineering from Çukurova University, Adana, Turkey, in 2009, the M.S. degree in biomedical engineering from Istanbul Technical University, Istanbul, Turkey, in 2010, and the M.S. and Ph.D. degrees in electricalelectronics engineering from Dicle University, Diyarbakır, Turkey, in 2012 and 2017, respectively.,From 2008 to 2009, he was an Exchange Student in electrical engineering from Linköping University, Sweden. He is currently an Assistant Professor of electrical-electronics engineering with Batman University and also the Head of the Electronics Division. His research interests include digital image processing, machine learning, and remote sensing applications.,Dr. Acar received awards and honors include The Scientific and Technological Research Council of Turkey (TUBITAK) Grant, Erasmus Mobility Grant, Sweden, and Erasmus Internship Grant, Germany.

Abdulkerim Öztekin, Berlin, Germany, in 1978. He received the B.S. degree in electrical and electronics engineering from Hacettepe University, Ankara, in 2001. He received the M.S. and the Ph.D. degrees in electrical and electronics engineering from Gaziantep University, Gaziantep, in 2018. From 2001 to 2011 , he worked as a Senior Engineer in several companies in the industry, and from 2012 to 2018; he was a Lecturer with the Electronic Communication Department, Batman University. Since 2018, he has been an Assistant Professor with the Electrical and Electronics Engineering Department, Batman University. His research interests include signal processing, video coding, communication systems, and machine learning.

Erdoğan Aldemir received the B.Sc. degree from Uludağ University in 2010 and the M.Sc. degree from the Ylldiz Technical University in 2013, all in electronics engineering. He received Ph.D. degree from Dokuz Eylül University in 2019. He is currently working on medical imaging, source coding, and image compression. 